\title{
Retroviral oncoprotein Tax targets coiled-coil centrosomal protein TAX1BP2 to induce centrosome overduplication
}

\author{
Yick-Pang Ching ${ }^{1,2}$, Shing-Fai Chan ${ }^{1, *}$, Kuan-Teh Jeang ${ }^{3}$ and Dong-Yan Jin ${ }^{1, \#}$ \\ ${ }^{1}$ Department of Biochemistry and ${ }^{2}$ Department of Pathology, Faculty of Medicine, The \\ University of Hong Kong, Hong Kong, China \\ ${ }^{3}$ Laboratory of Molecular Microbiology, National Institute of Allergy and Infectious \\ Diseases, 9000 Rockville Pike, Bethesda, MD 20892-0460, USA
}

*Current address: Center for Neuroscience and Aging, The Burnham Institute, 10901 North Torrey Pines Road, La Jolla, CA 92037, USA

\footnotetext{
\# Correspondence should be addressed to D.-Y. J. at: Department of Biochemistry, The University of Hong Kong, $3^{\text {rd }}$ Floor, Laboratory Block, Faculty of Medicine Building, 21 Sassoon Road, Hong Kong. Tel: +852-2819-9491; Fax: +852-2855-1254; E-mail: dyjin@hkucc.hku.hk
}

Data deposition: The human TAX1BP2 nucleotide sequence reported in this paper has been deposited in the GenBank database under accession number DQ139275. 
Emerging evidence suggests that supernumerary centrosomes drive genome instability and is linked to oncogenesis ${ }^{1-3}$. Human T-cell leukemia virus type I (HTLV-I) is etiologically associated with adult T-cell leukemia (ATL) ${ }^{4}$. ATL cells are aneuploid, but the causes of aneuploidy are incompletely understood ${ }^{5,6}$. Here we showed that centrosome amplification is frequent in HTLV-I-transformed cells and that this phenotype is caused by the viral Tax oncoprotein. We also found that the fraction of Tax protein which localizes to centrosomes interacts with TAX1BP2, a novel centrosomal protein composed almost entirely of coiled-coil domains. Overexpression of TAX1BP2 inhibited centrosome duplication, whereas depletion of TAX1BP2 by RNAi resulted in centrosome hyperamplification. Our findings suggest that HTLV-I Tax oncoprotein targets a novel centrosomal protein, TAX1BP2, to create genomic instability and aneuploidy. 
The centrosome is a major microtubule-organizing center that governs spindle assembly and bipolarity ${ }^{7}$. During the cell cycle it duplicates only once and undergoes characteristic changes. Impairment of the centrosome cycle may lead to chromosome missegregation and aneuploidy ${ }^{1}$. Indeed, centrosome abnormalities are frequent in various malignant tumors including chronic myeloid leukemia ${ }^{3}$. However, the role of centrosome disorder in adult T-cell leukemia (ATL), a fetal disease induced by a human retrovirus ${ }^{4}$, is not known.

ATL is caused by human T-cell leukemia virus type I (HTLV-I) ${ }^{4}$. Over 20 million individuals globally are infected with HTLV-I. This virus-induced leukemogenesis occurs in $2-5 \%$ of individuals over a latency period of more than 20 years ${ }^{6}$. Plausibly, for the infected cells to acquire selective growth advantages, HTLV-I has to initiate the disruption of several cellular events and surveillance mechanisms. How this occurs is not well understood; however, an HTLV-I-encoded oncoprotein, Tax, has been implicated in the immortalization and transformation of $\mathrm{CD}^{+} \mathrm{T}$-lymphocytes ${ }^{8}$. Tax is a transcriptional activator that has profound impact on cell signaling through the CRE, $\mathrm{KB}$ and $\mathrm{SRE}$ pathways and on the expression of various cytokines and proto-oncogenes ${ }^{5,8}$. In addition, Tax has also been shown to induce genome instability by disrupting several cell cycle checkpoints $^{5,10}$ leading to the accumulation of damaged cellular DNA ${ }^{11}$. For its various manifestations, current evidence indicates that Tax directly binds several discrete cellular proteins $^{5,8}$.

In viral transformation of cells, human papillomaviruses and hepatitis B virus induce centrosome pathology $y^{12,13}$. To ask if HTLV-I shares this aspect of oncogenesis, we investigated whether centrosomal abnormalities also occur in ATL cells. We visualized 
centrosomes in control Jurkat cells and two HTLV-I ${ }^{+}$ATL cell lines (MT4 and C816645) using anti- $\gamma$-tubulin antibody and quantified cells that had more than 2 centrosomes (Fig. 1a, b). Whereas $<5 \%$ of Jurkat cells had supernumerary centrosomes, the percentages of MT4 and C8166-45 cells with $>2$ centrosomes were significantly higher ( $30 \%$; see Fig. 1a, panel 2 for an example of MT4 cell with a cluster of centrosomes). These results provide the first evidence for centrosome amplification in ATL cells. We next asked whether the HTLV-1 Tax oncoprotein is causal for centrosome amplification. To address this question, we assayed centrosome duplication in a JPX9 cell line which carries a Tax gene driven by the inducible metallothionein promoter $^{10}$. JPX9 cells not induced for Tax showed cells with $>2$ centrosomes at a prevalence comparable to Jurkat cells (Fig. 1b, compare column 4 to 1). By contrast, induction of Tax expression in JPX9 cells led to a distinct increase in cells with supernumerary centrosomes (Fig. 1b, compare column 5 to 4). These results correlate Tax expression with centrosome amplification in $\mathrm{T}$ cells.

Because Jurkat, MT4 and C8166-45 leukemic cells might already have other hidden defects in cell cycle and growth control that contribute to the centrosome abnormalities induced by Tax, we sought to analyze centrosome duplication in IMR-90 primary human fibroblasts derived from normal fetal lung. Expression of Tax resulted in centrosome amplification in $\sim 30 \%$ of IMR-90 cells (Fig. 1b, compare column 7 to 6 ). Moreover, the supernumerary centrosomes in Tax-expressing IMR-90 cells contained centrobin (Fig. $1 b$, inset), a recently-identified coiled-coil protein preferentially associated with daughter centroles ${ }^{14}$. Thus, Tax alone is sufficient to induce centrosome duplication errors in normal human cells. 
To understand better the mechanism of Tax's effect on centrosomes, we performed overexpression assays in $\mathrm{CHO}$ cells. We used hydroxyurea (HU) to inhibit cellular DNA synthesis and uncouple centrosome duplication from DNA replication and cytokinesis, allowing for multiple rounds of centrosome duplication to occur within the cell ${ }^{15}$. We also employed the overexpression of $\mathrm{Cdk} 2$ and an $\mathrm{Rb}$ mutant lacking the $\mathrm{Cdk}$ phosphorylation sites $(\mathrm{Rb} \Delta \mathrm{Cdk})$. $\mathrm{Cdk} 2$ is a well-known stimulator of centrosome duplication ${ }^{15-17}$, and $\mathrm{Rb} \Delta \mathrm{Cdk}$ has been found to inhibit this process ${ }^{15}$. We found that $\mathrm{Cdk} 2$ and $\mathrm{Rb} \Delta \mathrm{Cdk}$ behaved in our assays as positive and negative regulators of centrosome duplication, respectively. We further observed that Tax stimulated centrosome duplication as effectively as Cdk2 (Fig. 1c, panel 2 compared to panel 1; and Fig. 1d, column 5 compared to columns 3 and 4). This is generally consistent with recent findings from another group ${ }^{18}$.

Tax localizes predominantly to nuclear and perinuclear speckles ${ }^{10,11}$; and this staining pattern is compatible with a fraction of Tax localizing to centrosomes. To shed light on how Tax might stimulate centrosome duplication, we investigated whether it could be found at centrosomes. We stained Tax-expressing HeLa cells simultaneously with antiTax and anti-pericentrin. We saw that while the majority of Tax was in the nucleus, a portion of Tax costained with pericentrin (Fig. 1e). To confirm this colocalization, we next immunoprecipitated extracts of HTLV-I-transformed MT4 cells with monoclonal anti- $\gamma$-tubulin antibody and queried by Western blotting for Tax in the immunoprecipitate. Tax did co-precipitate with $\gamma$-tubulin, an integral constituent of centrosomes, but it did not precipitate with material recovered using control irrelevant 
mouse IgG (Fig. 1f, lane 2 compared to lane 1). Taken together, the results support the presence of Tax at cellular centrosomes.

What component of the centrosome might Tax recognize? We had previously shown using yeast two-hybrid assay that Tax recognizes and interacts with several mammalian coiled-coil proteins ${ }^{19}$. These coiled-coil Tax-binding proteins include human mitotic checkpoint protein MAD $1^{10}$, IKB kinase regulatory subunit IKK $\gamma^{20}$, and another highly coiled-coil protein which we named TAX1BP2/TXBP121. TAX1BP2 has heretofore not been characterized but shares significant homology with centrosomal protein C-Nap ${ }^{21}$. We hypothesized that TAX1BP2 might be a centrosome constituent that tethers Tax to this organelle.

To confirm the interaction between TAX1BP2 and Tax, we raised rabbit polyclonal antisera against two synthetic TAX1BP2 peptides. The reactivity and specificity of these antibodies were verified by Western blotting and immunofluorescence (see examples in Fig. 2f). Using these antibodies and a monoclonal anti-Tax antibody, we assessed further the interaction between Tax and TAX1BP2 in cultured HeLa cells by reciprocal immunoprecipitations followed with immunoblotting. Hence, TAX1BP2 was detected in anti-Tax immunoprecipitates (Fig. 2a), and Tax in anti-TAX1BP2 precipitates (Fig. 2b). Next, we employed 7 TAX1BP2 mutants to map the Tax-binding domain in TAX1BP2 to amino acids 1109-1162 which contain a leucine zipper with four periodically spaced leucines. We noted that this Tax-binding domain is not conserved in TAX1BP2homologous centrosomal protein C-Nap1. When we mutated the leucine motif by replacing the second or the third leucine with proline, binding of Tax by TAX1BP2 was lost (Fig. 2c; mutants M5' and M5" compared to M5). The interaction between Tax and 
TAX1BP2-M5 (region 1109-1162) was also verified using GST pull-downs. We observed that recombinant His-Tax bound to GST-TAX1BP2 and GST-TAX1BP2-M5, but not to GST (Fig. 2d, compare lanes 2 and 3 to lane 1). In addition, we demonstrated the co-immunoprecipitation of Tax and TAX1BP2 from extracts of HTLV-I-transformed MT4 cells (Fig. 2e). Finally, inside intact HeLa and MT4 cells, Tax colocalized with TAX1BP2 at perinuclear dots expected for centrosomes (Fig. 2f).

A TAX1BP2-related protein is the $220-\mathrm{kD}$ CROCC protein which is the structural component of ciliary rootlet ${ }^{22,23}$. The mRNA of CROCC/rootletin shares a common 3' portion of the TAX1BP2 transcript (Fig. S1a). Further comparison of the two sequences revealed that TAX1BP2 and CROCC are alternatively spliced isoforms, likely transcribed from two different promoters. The retention of the $1.5-\mathrm{kb}$ intron 5 in TAX1BP2 leads to an alternate translational start site. CROCC is expressed abundantly in retina and sparsely in brain, trachea and kidney ${ }^{22}$. Consistent with this, we did not detect CROCC transcript in any of the human tissues and cells tested (data not shown). In contrast, we detected the specific expression of TAX1BP2 mRNA in various tissues and cells (Fig. S1b-d), and an $\sim 150-\mathrm{kD}$ TAX1BP2 protein was easily detected in HeLa cells (Fig. S1e).

TAX1BP2 is highly homologous to centrosomal protein C-Nap $1^{21}$, and the staining pattern of TAX1BP2 (Fig. 2f) is generally compatible with that of centrosome. Next, we directly confirmed that TAX1BP2 was present at centrosomes by demonstrating colocalization of cell endogenous TAX1BP2 (Fig. 3a) and exogenously overexpressed HAtagged TAX1BP2 (Fig. 3b) with $\gamma$-tubulin and pericentrin (data not shown). Further, we characterized in TAX1BP2 two separate centrosome-targeting domains corresponding to 
amino acids 1-941 (truncated mutant M6) and 1040-1313 (mutant M7) (Fig. 3c, d). These domains when fused to GFP are sufficient to target the heterologous GFP protein to the centrosome (Fig. 3d and data not shown). Co-immunoprecipitation experiment also verified that the GFP-TAX1BP2-M7 protein was in a protein complex containing $\gamma$ tubulin (Fig. 3f). Interestingly, the centrosomal localization of TAX1BP2 is independent of the integrity of microtubules, because treatment with either microtubule-polymerizing agent taxol (Fig. 3e) or microtubule-disrupting agent nocodazole (data not shown) did not perturb its localization.

The centrosomal localization of TAX1BP2 (Fig. 3), its interaction with Tax (Fig. 2), and the induction by Tax of supernumerary centrosomes (Fig. 1) prompted us to consider a role for TAX1BP2 in governing centrosome duplication. To test this hypothesis, we overexpressed TAX1BP2 in CHO cells and assessed centrosome duplication. Intriguingly, the number of cells with supernumerary centrosomes in TAX1BP2overexpressing cells was significantly lower than in either control cells or cells overexpressing Cdk2 (Fig. 4a). This apparent inhibitory effect of TAX1BP2 is comparable to the repression of centrosome duplication previously reported for $\mathrm{Rb} \Delta \mathrm{Cdk}^{15}$. The inhibition was specifically induced by TAX1BP2 and was not observed with TAX1BP2-homologous centrosomal protein C-Nap ${ }^{21}$, Tax-binding cell cycle regulatory protein $\mathrm{MAD}^{20}$, or a dominant inactive mutant of $\mathrm{MAD}^{20}$ (MAD1M4; Fig. 4a, columns 6-9 compared to column 5). Because the phenotype based on overexpression of transfected TAX1BP2 cannot formally comment on the role of cell endogenous TAX1BP2, we next performed knock-down experiments. We designed two small hairpin RNAs (shRNAs; shBP2a and shBP2b) targeting coding sequences of TAX1BP2 
transcript and transfected them into $\mathrm{CHO}$ cells. While shBP2a was more effective in depleting TAX1BP2, both shRNAs showed suppressive activities (Fig. 4b, lanes 2, 3, 5 and 6 compared to lane 1). When separately expressed, both shBP2a and shBP $2 b$ moderately enhanced the number of cells with supernumerary centrosomes (Fig. 4c, columns 4 and 5 compared to column 2), suggesting that the depletion of endogenous TAX1BP2 removed a negative effect on centrosome duplication. In keeping with the concept that Tax might function through inhibition of TAX1BP2, expression of Tax did not further stimulate centrosome amplification in TAX1BP2-depleted cells (Fig. 4c, column 6 compared to column 4). When we omitted treatment of cells with HU and propagated cells after transfection for $72 \mathrm{~h}$, the effects of TAX1BP2 knock-down became more evident. Thus, the percentage of shRNA-transfected cells with increased centrosomes became significantly higher than those from control GFP- and TAX1BP2expressing cells not treated with HU (Fig. 4d, columns 4 and 5 compared to columns 1 and 2; and Fig. S2, panels 6 and 9 compared to panel 3). Again, while Tax sufficiently induced centrosome duplication in CHO cells, Tax could not further enhance the effect induced by depletion of TAX1BP2 (Fig. 4d, column 6 compared to columns 3 and 4). Altogether, the results from both exogenous overexpressions and endogenous knockdowns support a repressive role for TAX1BP2 in centrosome duplication, which is likely targeted by Tax.

Above data show that Tax and TAX1BP2 have opposing effects on centrosome duplication. We next formally addressed if TAX1BP2 is the centrosomal protein targeted through direct protein-protein binding by Tax. If this reasoning is correct, then one prediction is that overexpression of TAX1BP2 should blunt Tax's capacity to induce 
supernumerary centrosomes. We found that TAX1BP2 did effect a dose-dependent reduction in the number of Tax-expressing cells with amplified centrosomes (Fig. 5a, columns 5 and 6 compared to column 4). Conversely, increased overexpression of Tax overcame the block to centrosome amplification by TAX1BP2 (Fig. 5a, columns 7 and 8 compared to column 3). In contrast, coexpression with TAX1BP2 of a Tax point mutant (S132A) lacking the ability to interact with $\mathrm{TAX} 1 \mathrm{BP} 2^{19}$ had no influence on the inhibition of centrosome duplication (Fig. 5, column 9 compared to column 3).

We next assessed the centrosome phenotype of this Tax mutant and found that it was able to activate the HTLV-I LTR in a manner comparable to wild type Tax (Fig. 5b, column 3 compared to column 2), but it was no longer able to induce the centrosome amplification seen with wild type Tax (Fig. 5c, column 4 compared to columns 2 and 3). Thus, the interaction with TAX1BP2 correlates with Tax induction of centrosome overduplication. Importantly, this Tax mutant also lost the ability to transform rat embryonic fibroblasts (Fig. 5d) or to induce micronuclei formation (Fig. 5e), a marker for genome instability caused by the expression of $\operatorname{Tax}^{24}$. Mechanisms by which Tax induces transformation and genome instability are complex ${ }^{5,8}$. The correlation of Tax-TAX1BP2 interaction with cellular transformation and genome instability does not exclude the involvement of other Tax-binding proteins. However, one interpretation to our findings is that the interaction with TAX1BP2 might serve one contributory role in these processes.

On the other hand, we noted that a TAX1BP2 mutant (M6) which was fully repressive for centrosome duplication (Fig. 5f, column 4 compared to column 3) but does not contain the Tax-binding domain (Fig. 2c), was unable to rescue fully the phenotype of Tax (Fig. 5f, column 7 compared to column 6). Likewise, another TAX1BP2 mutant 
(M4) defective in centrosomal targeting had no influence on the stimulatory effect of Tax (Fig. 5f, column 9 compared to column 5). Collectively, these results are consistent with the disruptive effect of Tax on centrosome amplification being mediated through direct protein-protein binding to TAX1BP2. What currently remains unanswered and needs further investigation is the mechanism for the repressive effect of TAX1BP2 on centrosome duplication.

Here, we report on the finding of frequent amplification of centrosomes in cells expressing the HTLV-I Tax oncoprotein (Fig. 1). We suggest that Tax induces excessive centrosome duplication (Fig. 5) by targeting a hitherto novel centrosomal protein TAX1BP2 (Fig. 2 and Fig. 5). Our evidence supports that TAX1BP2 may function to block centrosome reduplication (Fig. 3 and Fig. 4). Unlike centriolin and other centrosomal proteins that are also required for cytokinesis ${ }^{25}$, TAX1BP2 was not found in the midbody and depletion of TAX1BP2 did not lead to the formation of multinuclear syncytia (Fig. 4). Based on sequence homology and similarity in staining patterns, TAX1BP2 is more closely related to centrosomal coiled-coil proteins that include C$\mathrm{Nap1}^{21}$, CEP110 and ninein ${ }^{26}$. C-Nap1 is the substrate of Nek2 kinase, a key regulator of centrosome structure and function ${ }^{21}$. On the other hand, Cdk2 kinase has been shown to regulate centrosome duplication partly through phosphorylation of nucleophosmin ${ }^{27}$. Thus, it will be of interest to investigate whether TAX1BP2 is phosphorylated by Nek2 or Cdk2. In this regard, one recent study has demonstrated the centrosomal localization and function of CROCC as well as the phosphorylation of CROCC by Nek2 ${ }^{28}$. In addition, an interaction between CROCC and C-Nap1 has also been shown with yeast two-hybrid assay ${ }^{28}$. While we were able to detect, by co-immunoprecipitation and yeast 
two-hybrid analysis, the homodimerization of TAX1BP2 (Fig. S3) and the interaction between TAX1BP2 and C-Nap1 (data not shown), the modification of TAX1BP2 was not seen in HeLa cells transfected with active Nek2 (Fig. S4a, lane 3 compared to lane 2). Neither could we observe the phosphorylation of TAX1BP2 by Nek2 in vitro (data not shown). In contrast, we detected Cdk2 phosphorylation of TAX1BP2 both in Cdk2overexpressing cells (Fig. S4b) and by in vitro kinase assay (Fig. S4c).

Because the molecular mechanisms through which centrosome duplication is regulated are not well understood, it may be that TAX1BP2 is a component of the previously identified centrosome-intrinsic machinery that blocks reduplication ${ }^{29}$. In line with this hypothesis, the phosphorylation of TAX1BP2 by Cdk2 (Fig. S4) suggests that TAX1BP2 activity is regulated in a cell cycle-specific manner. Future analysis of how TAX1BP2 prevents centrosome overduplication and how Tax interferes with this function will reveal new aspects to the cellular control of genome stability and viral strategies to subvert this control.

Whether centrosome abnormalities are the cause or consequence of tumor initiation and progression remains hotly debated ${ }^{3,30}$. HTLV-1 Tax is a well-studied transforming protein $^{5,8}$. Our finding that Tax targets a centrosomal component to subvert cellular control of centrosome replication supports the notion that disordered centrosome amplification has a contributory role in driving tumorigenesis. Data here are consistent with previous findings from human papillomaviruses ${ }^{12}$ and hepatitis $B$ virus ${ }^{13}$ and suggest that dysregulation of normal centrosome metabolism may be a transforming mechanism conserved by diverse tumor viruses. 


\section{METHODS}

Cells. Human Jurkat and JPX9 T lymphocytes and HTLV-I ${ }^{+}$MT4 and C8166 cells were grown in RPMI 1640 medium containing 10\% fetal bovine serum, glutamate and antibiotics. Human normal fetal lung fibroblasts IMR-90, human cervical carcinoma cell line $\mathrm{HeLa}$, Chinese hamster ovary cell line $\mathrm{CHO}$, African green monkey kidney cell line COS7 and rat embryonic fibroblasts REF were maintained in supplemented Dulbecco's modified Eagle's medium. IMR-90, HeLa, CHO and REF cells were transfected using LipofectAMINE $2000^{\mathrm{TM}}$ reagent (Invitrogen).

Plasmids and antibodies. Full-length TAX1BP2 cDNA clone (KIAA0445) was kindly provided by Dr. Takahiro Nagase (Kazusa DNA Research Institute, Japan). cDNA for CNap1 was a gift from Dr. Jerome Rattner (University of Calgary, Canada). Expression plasmid for $\gamma$-tubulin-GFP ${ }^{31}$ was supplied by Dr. Alexey Khodjakov (Wadsworth Center, New York, USA). cDNA for Nek2 was amplified from I.M.A.G.E. clone 3597227 obtained from RZPD Deutsches Ressourcenzentrum für Genomforschung GmbH (Berlin, Germany). Expression vector pHA, pLTR-fLuc reporter plasmid, as well as expression plasmids for Tax, TaxM, Cdk2, MAD1 and MAD1M4 have been described elsewhere ${ }^{9,10,20}$. Expression vectors pFLAG-CMV2 and pEGFP were from Eastman Kodak and Clontech, respectively. $\mathrm{Rb} \Delta \mathrm{Cdk} 2$ expression plasmid ${ }^{15}$ was a gift from Dr. Jiri Bartek (Institute of Cancer Biology, Denmark). Mouse monoclonal anti- $\gamma$-tubulin (clone GTU-88), rabbit polyclonal anti- $\gamma$-tubulin (T3559), mouse monoclonal anti- $\alpha$ tubulin (clone B-5-1-2), and mouse monoclonal anti-FLAG (clone M5) antibodies were from Sigma. Rabbit polyclonal anti-centrobin antibody ${ }^{14}$ was kindly provided by Dr. 
Qingshen Gao (Northwestern University, Illinois, USA). Rabbit polyclonal antipericentrin was from BAbCo-Covance. Mouse monoclonal anti-HA (clone F-7) and rabbit polyclonal anti-GFP (sc-8334) were from Santa-Cruz. Mouse monoclonal anti-Tax (clone 18A51-42) and rabbit polyclonal anti-Tax have been described elsewhere ${ }^{10,20}$. Polyclonal antiserum $\alpha-121 \mathrm{a}$ and $\alpha-121 \mathrm{~b}$ were raised rabbits, against synthetic TAX1BP2 peptides LQAEKAEV AEALTKAEAGRVE LEL (amino acids 2-25) and TERTLEARERAHRQRVSTLKG (amino acids 1271-1291), respectively.

Centrosome duplication assay. Centrosome duplication assay was performed in $\mathrm{CHO}$ cells as described ${ }^{15}$. Briefly, cells were seeded at low density and treated with $4 \mathrm{mM} \mathrm{HU}$ for $40 \mathrm{~h}$. The number of centrosomes in 100-200 cells was analyzed by staining $\gamma$-tubulin. Transfected cells were identified by staining for the overexpressed protein or for GFP marker protein. Three to six independent experiments were usually performed for each transfection.

Protein analysis and luciferase reporter assay. Co-immunoprecipitation, GST pulldown, yeast two-hybrid analysis, Western blotting, and in vitro kinase assay with recombinant Cdk2 and Nek2 were performed as previously described ${ }^{10,20,32,33}$. Dualluciferase assay was carried out as per protocols recommended by Promega.

Confocal microscopy. Jurkat, JPX9, MT4 and C8166 cells were adsorbed to poly-Llysine-coated coverslips, while HeLa and CHO cells were directly cultured on coverslips. 
Cells were fixed with cold methanol or with $4 \%$ paraformaldehyde $(\mathrm{pH} 7.4)$ in PBS. The coverslips with attached cells were mounted on glass slides using VECTORSHIELD agent (VECTOR). Multi-color immunofluorescence imaging was then performed on a Zeiss LSM510 laser scanning confocal microscope.

RNAi knockdown of TAX1BP2. The shRNA expression vector pSHAG- $1^{34}$ was a kind gift from Dr. Greg Hannon (Cold Spring Harbor Laboratory, New York, USA). The shRNA expression cassettes containing U6 promoter-sense-loop-antisense-termination signal $\mathrm{T}_{6}$ were amplified by PCR and inserted into the vector through TA cloning. To knockdown the expression of TAX1BP2, the expression vector for shRNA against TAX1BP2 $(1 \mu \mathrm{g}$ or $2 \mu \mathrm{g})$ was transfected into cells. Two shRNAs shBP2a and shBP2b, which target nucleotides 1776-1796 and 3236-3256, respectively, of the TAX1BP2 mRNA transcript, were designed and used in the knockdown experiment.

Focus formation assay and micronuclei formation assay. For focus formation assay $^{35,36}$, REF cells were transfected with expression vectors for Tax and activated Ras (RasV12). The number of foci was scored after 21 days. Tax induction of micronuclei was measured in $\operatorname{COS7}$ cells as previously described ${ }^{24}$. 


\section{Acknowledgements}

We thank K.-T. Chin, A.C.S. Chun and Y.T. Siu for critical reading of the manuscript. This work was supported by a grant to D.-Y. J. (HKU 7249/01M) from the Hong Kong Research Grants Council. D.-Y. J. is a Leukemia and Lymphoma Society Scholar and a recipient of National Institutes of Health GRIP New Foreign Investigator Award (R01 TW06186-01) and National Natural Science Foundation of China Young Investigator Award (30029001). 


\section{References}

1. D'Assoro, A. B., Lingle, W. L. \& Salisbury, J. L. Centrosome amplification and the development of cancer. Oncogene 21, 6146-6153 (2002).

2. Pihan G. A., Purohit A., Wallace J., Knecht H., Woda B., Quesenberry P. \& Doxsey S. J. Centrosome defects and genetic instability in malignant tumors. Cancer Res. 58, 3974-3985 (1998).

3. Kramer, A. Centrosome aberrations - hen or egg in cancer initiation and progression? Leukemia 19, 1142-1144 (2005).

4. Gallo, R. C. The discovery of the first human retrovirus: HTLV-1 and HTLV-2. Retrovirology 2, 17 (2005).

5. Jeang, K.-T., Giam, C.-z., Majone, F. \& Aboud, M. Life, death, and Tax: role of HTLV-I oncoprotein in genetic instability and cellular transformation. J. Biol. Chem. 279, 31991-31994 (2004).

6. Matsuoka, M. \& Jeang, K.-T. Human T-cell leukemia virus type I at age 25: a progress report. Cancer Res. 65, 4467-4470 (2005).

7. Doxsey, S., McCollum, D. \& Theurkauf, W. Centrosomes in cellular regulation. Annu. Rev. Cell Dev. Biol. 21, 411-434 (2005).

8. Gatza, M. L., Eatt, J. C \& Marriott, S. J. Cellular transformation by the HTLV-I Tax protein, a jack-of-all-trades. Oncogene 22, 5141-5149 (2003).

9. Ching, Y.-P., Chun, A.C.S., Chin, K.-T., Zhang, Z.-Q., Jeang, K.-T. \& Jin, D.-Y. Specific TATAA and bZIP requirements suggest that HTLV-I Tax has transcriptional activity subsequent to the assembly of an initiation complex. Retrovirology 1, 18 (2004).

10. Jin, D.-Y., Spencer, F. \& Jeang, K.-T. Human T-cell leukemia virus type I oncoprotein Tax targets the human mitotic checkpoint protein MAD1. Cell 93, 8191 (1998). 
11. Haoudi, A., Daniels, R.C., Wong, E., Kupfer, G. \& Semmes, O.J. Human T-cell leukemia virus-I tax oncoprotein functionally targets a subnuclear complex involved in cellular DNA damage-response. J. Biol. Chem. 278, 37736-37744 (2003).

12. Duensing, S. \& Munger, K. Human papillomaviruses and centrosome duplication errors: modeling the origins of genomic instability. Oncogene 21, 6241-6248 (2002).

13. Forgues, M., Difilippantonio, M. J., Linke, S. P., Ried, T., Nagashima, K., Feden, J., Valerie, K., Fukasawa, K. \& Wang, X. W. Involvement of Crm1 in hepatitis B virus $\mathrm{X}$ protein-induced aberrant centriole replication and abnormal mitotic spindles. Mol. Cell. Biol. 23, 5282-5292 (2003).

14. Zou, C., Li, J., Bai, Y., Gunning, W.T., Wazer, D.E., Band, V. \& Gao, Q. Centrobin: a novel daughter centriole-associated protein that is required for centriole duplication. J. Cell Biol. 171, 437-445 (2005).

15. Meraldi, P., Lukas, J., Fry, A. M., Bartek, J. \& Nigg, E. A. Centrosome duplication in mammalian somatic cells requires E2F and Cdk2-cyclin A. Nature Cell Biol. 1, 88-93 (1999).

16. Lacey, K.R., Jackson, P.K. \& Stearns, T. Cyclin-dependent kinase control of centrosome duplication. Proc. Natl. Acad. Sci. USA 96, 2817-2822 (1999).

17. Matsumoto, Y., Hayashi, K. \& Nishida, E. Cyclin-dependent kinase 2 (Cdk2) is required for centrosome duplication in mammalian cells. Curr. Biol. 9, 429-432 (1999).

18. Peloponese, J.M. Jr., Haller, K., Miyazato, A. \& Jeang, K.T. Abnormal centrosome amplification in cells through the targeting of Ran-binding protein-1 by the human $\mathrm{T}$ cell leukemia virus type-1 Tax oncoprotein. Proc. Natl. Acad. Sci. USA 102, 18974-18979 (2005). 
19. Chun, A.C.S., Zhou, Y., Wong, C.-M., Kung, H.-F., Jeang, K.-T. \& Jin, D.Y. Coiled-coil motif as a structural basis for the interaction of HTLV-I Tax with cellular cofactors. AIDS Res. Hum. Retrov. 16, 1689-1694 (2000).

20. Jin, D.-Y., Teramoto, H., Giam, C.-Z., Chun, R.F., Gutkind, J.S. \& Jeang, K.-T. A human suppressor of c-Jun N-terminal kinase 1 activation by tumor necrosis factor a. J. Biol. Chem. 272, 25816-25823 (1997).

21. Fry, A. M., Mayor, T., Meraldi, P., Stierhof, Y.-D., Tanaka, K. \& and Nigg, E. A. C-Nap1, a novel centrosomal coiled-coil protein and candidate substrate of the cell cycle-regulated protein kinase Nek2. J. Cell Biol. 141, 1563-1574 (1998).

22. Yang, J., Liu, X., Yue, G., Adamian, M., Bulgakov, O. \& Li, T. Rootletin, a novel coiled-coil protein, is a structural component of the ciliary rootlet. J. Cell Biol. 159, 431-440 (2002).

23. Yang, J., Gao, J., Adamian, M., Wen, X.H., Pawlyk, B., Zhang, L., Sanderson, M. J., Zuo, J., Makino, C. L. \& Li, T. The ciliary rootlet maintains long-term stability of sensory cilia. Mol. Cell. Biol. 25, 4129-4137 (2005).

24. Semmes, O.J., Majone, F., Cantemir, C., Turchetto, L., Hjelle, B. \& Jeang, K.T. HTLV-I and HTLV-II Tax: differences in induction of micronuclei in cells and transcriptional activation of viral LTRs. Virology 217, 373-379 (1996).

25. Gromley, A., Jurczyk, A., Sillibourne, J., Halilovic, E., Mogensen, M., Groisman, I., Blomberg, M. \& Doxsey, S. A novel human protein of the maternal centriole is required for the final stages of cytokinesis and entry into S phase. J. Cell Biol. 161, 535-545 (2003).

26. Ou, Y.Y., Mack, G. J., Zhang, M. \& Rattner, J. B. CEP110 and ninein are located in a specific domain of the centrosome associated with centrosome maturation. J. Cell Sci. 115, 1825-1835 (2002).

27. Okuda, M. Horn, H. F., Tarapore, P., Tokuyama, Y., Smulian, A. G., Chan, P. K., Knudsen, E. S., Hofmann, I. A., Snyder, J. D., Bove, K. E. \& Fukasawa, K. Nucleophosmin/B23 is a target of CDK2/cyclin E in centrosome duplication. Cell 103, 127-140 (2000). 
28. Bahe, S., Stierhof, Y.-D., Wilkinson, C. J., Leiss, F. \& Nigg, E. A. Rootletin forms centriole-associated filaments and functions in centrosome cohesion. J. Cell Biol. 171, 27-33 (2005)

29. Wong, C. \& Stearns, T. Centrosome number is controlled by a centrosome-intrinsic block to reduplication. Nat. Cell Biol. 5, 539-544 (2003).

30. Nigg, E. A. Centrosome aberrations: cause or consequence of cancer progression? Nat. Rev. Cancer. 2, 815-825 (2002).

31. Khodjakov, A. \& Rieder, C.L. The sudden recruitment of $\gamma$-tubulin to the centrosome at the onset of mitosis and its dynamic exchange throughout the cell cycle, do not require microtubules. J. Cell Biol. 146, 585-596 (1999).

32. Chin, K.-T., Zhou, H.-J., Wong, C.-M., Lee, J.M.-F., Chan, C.-P., Qiang, B.-Q., Yuan, J.-G., Ng, I.O.-L. \& Jin, D.-Y. The liver-enriched transcription factor CREB$\mathrm{H}$ is a growth suppressor protein underexpressed in hepatocellular carcinoma. Nucl. Acids Res. 33, 1859-1873 (2005).

33. Ching, Y.P., Pang, A.S., Lam, W.H., Qi, R.Z. \& Wang, J.H. Identification of a neuronal Cdk5 activator-binding protein as Cdk5 inhibitor. J. Biol. Chem. 277, $15237-15240$ (2003)

34. Paddison, P.J., Caudy, A.A. \& Hannon, G.J. Stable suppression of gene expression by RNAi in mammalian cells. Proc. Natl. Acad. Sci. USA 99, 1443-1448 (2002).

35. Jin, D. Y., Wang, H. L., Zhou, Y., Chun, A.C.S., Kibler, K. V., Hou, Y. D., Kung, H. \& Jeang, K. T. Hepatitis C virus core protein-induced loss of LZIP function correlates with cellular transformation. EMBO J. 19, 729-740 (2000)

36. Ching, Y.P., Wong, C.M., Chan, S.F., Leung, T.H., Ng, D.C.H., Jin, D.Y. \& Ng, I.O.L. Deleted in liver cancer (DLC) 2 encodes a RhoGAP protein with growth suppressor function and is underexpressed in hepatocellular carcinoma. J. Biol. Chem. 278, 10824-10830 (2003) 


\section{FIGURE LEGENDS}

Figure 1 Centrosome amplification in HTLV-I-transformed and Tax-expressing cells. (a) Centrosome staining of Jurkat (Jkt) and MT4 cells. HTLV-I- Jurkat T lymphocytes and HTLV-I ${ }^{+}$MT4 cells were loaded onto poly-L-lysine-coated coverslips and stained for $\gamma$ tubulin. Bar, $30 \mu \mathrm{m}$. (b) Quantitation of cells with $>2$ centrosomes. Numbers of cells with $>2$ centrosomes were counted individually from 100 of Jurkat, MT4, C8166-45, uninduced JPX9, induced JPX9 (JPX9i), IMR-90, and Tax-expressing IMR-90 (IMR90x) cells. JPX9i cells were treated with $25 \mu \mathrm{m} \mathrm{CdSO}_{4}$ for $3 \mathrm{~h}$ to induce the expression of Tax. Some IMR-90x cells were co-transfected with an expression plasmid for $\gamma$-tubulinGFP. Centrosomes were either stained with anti- $\gamma$-tubulin (Sigma) or identified by $\gamma$ tubulin-GFP fluorescence. Daughter centrioles in IMR-90x cells were stained with anticentrobin and a representative image of multiple daughter centrioles in one cell was shown in the inset. (c) Centrosome duplication assay in hydroxyurea (HU)-treated $\mathrm{CHO}$ cells. Cells were transfected with $\mathrm{Rb} \Delta \mathrm{Cdk}(\mathrm{Rb} \Delta)$ and $\mathrm{Tax}$ expression plasmids, respectively. Cells were treated with $4 \mathrm{mM} \mathrm{HU}$ for $40 \mathrm{~h}$ (except for group 1, which is the no HU control) and transfected cells (highlighted with arrow) were identified by staining for Tax or Rb. Centrosomes were stained with anti- $\gamma$-tubulin (Sigma). The inset on the top of panel 2 is a portion of the image shown at higher magnification which illustrates more clearly the amplified centrosomes in Tax-expressing cells. (d) Quantitative analysis of centrosome numbers in cells transfected with the variously indicated expression plasmids. (e) Centrosomal localization of Tax. HeLa cells were transfected with Tax expression plasmid and stained for Tax (panel 1) and pericentrin (panel 2). In panel 3, Green (representing Tax) and red (representing pericentrin) fluorescent signals were 
overlaid. Colocalizations are shown in yellow. Arrows point to centrosome signals in transfected cells. (f) Tax association with $\gamma$-tubulin. Extracts of MT4 and Jurkat cells were immunoprecipitated with mouse $\operatorname{IgG}$ or mouse anti- $\gamma$-tubulin $(\alpha-\gamma$-tub). The precipitates were then Western blotted with anti-Tax. An uncropped image of the blot is shown in Fig. S5. Results in $\mathbf{b}$ and $\mathbf{d}$ represent mean \pm standard deviation from three independent experiments.

Figure 2 Interaction between Tax and TAX1BP2. (a, b) Co-immunoprecipitation of Tax and TAX1BP2 from Tax-expressing cells. HeLa cells were transfected with a Tax expression plasmid. Reciprocal immunoprecipitation and Western blotting were performed with mouse anti-Tax ( $\alpha$-Tax) and rabbit anti-TAX1BP2 ( $\alpha$-BP2). (c) Taxbinding domain in TAX1BP2. Interactions between TAX1BP2 mutants and Tax, as indicated by + (interaction) or - (lack of interaction), were analyzed by yeast two-hybrid assay. In mutants M5' and M5', a leucine in the leucine zipper motif was replaced by a proline using site-directed mutagenesis. (d) GST pull-down assay. His-tagged Tax, GST, GST-TAX1BP2 (BP2) and GST-TAX1BP2-M5 (M5) proteins were produced in E. coli. His-Tax was then equilibrated individually with GST, GST-TAX1BP2 and GSTTAX1BP2-M5 resins. Proteins bound to the resins were analyzed by Western blotting with anti-His antibodies. (e) Co-immunoprecipitation of Tax and TAX1BP2 from HTLVI-transformed MT4 cells. Immunoprecipitation and Western blotting were performed with extracts of MT4 cells as in b. (f) Colocalization of Tax and TAX1BP2. HeLa cells transfected with Tax expression plasmid and MT4 cells were stained for Tax (panels 1 
and 4) and TAX1BP2 (BP2; panels 2 and 5). Green (representing Tax) and red (representing TAX1BP2) fluorescent signals were then merged (panels 3 and 6) and colocalizations are in yellow (highlighted by arrows). The centrosomal staining pattern of Tax is representative of 61-74 \% Tax-expressing cells. Colocalization of Tax with TAX1BP2 at the centrosomes was observed in $85 \%$ of cells exhibiting centrosome amplification. Bar, $30 \mu \mathrm{m}$. Uncropped images of the blots in $\mathbf{d}$ and $\mathbf{e}$ are presented in Fig. S5.

Figure 3 TAX1BP2 is a centrosomal protein. (a) Colocalization of endogenous TAX1BP2 and $\gamma$-tubulin in HeLa cells. (b) Colocalization of overexpressed HA-tagged TAX1BP2 (HA-BP2) and $\gamma$-tubulin in HeLa cells. (c) Centrosomal localization of TAX1BP2-M6 in HeLa cells. (d) TAX1BP2-M7 is sufficient to target GFP to a microtubule-organizing center. HeLa cells were transfected with GFP-TAX1BP2-M7 and stained with anti-GFP (panel 1) and anti- $\alpha$-tubulin (panel 2). (e) Microtubuleindependent localization of TAX1BP2 to the centrosome. HeLa cells transfected with an HA-TAX1BP2 expression plasmid were treated with $1 \mathrm{ng} / \mathrm{ml}$ taxol for $16 \mathrm{~h}$ and stained for HA-TAX1BP2 (panel 1) and $\alpha$-tubulin (panel 2). (f) Association of GFP-TAX1BP2M7 with $\gamma$-tubulin. HeLa cells were transfected with GFP-TAX1BP2-M7 expression plasmid (lane 2) and immunoblotted with anti-GFP and anti- $\gamma$-tubulin antibodies (lanes 1 and 2). Extracts of transfected cells were immunoprecipitated (i.p.) with anti-GFP ( $\alpha$ GFP) or rabbit IgG. The precipitates were then probed with anti- $\gamma$-tubulin (lanes 3-5). An uncropped image of the blot is presented in Fig. S5. 
Figure 4 TAX1BP2 inhibits centrosome duplication. (a) Suppression of centrosome duplication by overexpression of TAX1BP2. CHO cells were transfected with indicated expression plasmids. Cells were treated with $4 \mathrm{mM} \mathrm{HU}$ for $40 \mathrm{~h}$ (except for group 1) and transfected cells (100 cells in each group) were quantitatively analyzed by staining for $\gamma$ tubulin. Percentages of cells with $>2$ centrosomes were plotted. (b) Depletion of TAX1BP2 by RNAi. Two shRNAs targeting TAX1BP2 coding sequences were expressed from DNA vector pSHAG-1 in CHO cells expressing HA-tagged TAX1BP2. Two different doses of shRNA plasmid were used and the expression of HA-TAX1BP2 and $\alpha$-tubulin in transfected cells were examined by Western blotting. Uncropped images of the blots are shown in Fig. S5. (c) Centrosome duplication assay in TAX1BP2depleted cells. CHO cells were co-transfected with EGFP plasmid and shRNAs targeting TAX1BP2, treated with HU (except for group 1), and analyzed for centrosome duplication. shRNA-transfected cells were identified by GFP fluorescence. (d) Centrosome hyperamplification in TAX1BP2-depleted cells. CHO cells co-transfected with GFP plus TAX1BP2, Tax or TAX1BP2-depleting shRNAs were incubated for $72 \mathrm{~h}$ and the numbers of centrosomes were determined by $\gamma$-tubulin staining. shBP2expressing cells were also sorted out by GFP fluorescence and subjected to flow cytometric analysis of DNA content. Cells with $4 \mathrm{~N}$ or $>4 \mathrm{~N}$ DNA content were not found upon depletion of TAX1BP2 (data not shown). Neither was cleavage failure found in TAX1BP2-depleted cells as stained with anti- $\alpha$-tubulin. Results are representative of mean \pm standard deviation from six independent experiments. 
Figure 5 Tax induction of centrosome overduplication is through targeting of TAX1BP2. (a) Overexpression of TAX1BP2 counteracts the influence of Tax on centrosome duplication. $\mathrm{CHO}$ cells were transfected with indicated expression plasmids. Cells were treated with $4 \mathrm{mM} \mathrm{HU}$ for $40 \mathrm{~h}$ (except for group 1) and transfected cells (100 in each group) were quantified for $>2$ centrosomes. Fixed amount of Tax plasmid plus increasing amounts of TAX1BP2 plasmid (columns 5 and 6), fixed amount of TAX1BP2 plus increasing amounts of Tax (columns 7 and 8), and TAX1BP2 plus the highest dose of Tax mutant S132A (TaxM; column 9) were used for transfection. (b) Transcriptional activity of Tax mutant. CHO cells were co-transfected with luciferase reporter plasmid pLTR-fLuc under the control of HTLV-I LTR and expression plasmid for either Tax or TaxM. Dual luciferase assays were performed and the relative firefly luciferase activity was obtained by normalizing to the readouts of Renilla luciferase. (c) TaxM lacks the ability to bind TAX1BP2 and does not stimulate centrosome duplication. (d) TaxM does not induce focus formation in rat embryonic fibroblasts in cooperation with Ras. (e) TaxM does not induce the formation of micronuclei in COS7 cells. (f) TAX1BP2-M6 lacks the ability to bind Tax and does not counteract Tax stimulation of centrosome duplication. Results represent mean \pm standard deviation from six independent experiments.

Figure S1 Expression of TAX1BP2 mRNA and protein in human tissues and cells. (a) Schematic diagrams of isoform-specific exons in TAX1BP2 and CROCC. Exons with 
identical sequences are shown with the same color. (b, c) Northern blot analyses of human cancer cell lines and tissues. A TAX1BP2-specific probe was used and TAX1BP2 transcripts are arrowed. (d) RT-PCR analyses of TAX1BP2 mRNA. A pair of TAX1BP2specific primers was used to detect TAX1BP2 mRNA in a group of human cancer cell lines. TAX1BP2 and $\beta$-actin transcripts are highlighted. (e) Western blot analysis of endogenous and overexpressed TAX1BP2 in HeLa cells. HeLa cells (lane 1) and HeLa cell transfected with TAX1BP2-expressing plasmid (lane 2; HeLa/BP2) were probed with anti-TAX1BP2 antiserum. TAX1BP2 protein of $\sim 150 \mathrm{kD}$ is arrowed.

Figure S2 Depletion of TAX1BP2 induces centrosome hyperamplification. CHO cells were co-transfected with GFP plus TAX1BP2-depleting shRNAs (shBP2a and shBP2b). Cells were incubated for $72 \mathrm{~h}$ and the numbers of centrosomes were determined by $\gamma$ tubulin staining (panels 2, 5 and 8). Transfected cells were identified by GFP staining (panels 1, 4 and 7). Bar, $30 \mu \mathrm{m}$.

Figure S3 Homodimerization of TAX1BP2. (a) Co-immunoprecipitation. HeLa cells were co-transfected with FLAG-tagged TAX1BP2 (fBP2) and HA-tagged TAX1BP2 (haBP2) expression plasmids, and immunoblotted with anti-HA (upper panel). Extracts of transfected cells were immunoprecipitated with anti-HA. The precipitates were then probed with anti-FLAG (lower panel). (b) Mapping of dimerization domain in TAX1BP2. Interactions between TAX1BP2 mutants and TAX1BP2, as indicated by + (interaction) or - (lack of interaction), were analyzed by yeast two-hybrid assay. The dimerization 
domain maps to amino acids 1137-1313, very close to the Tax-binding domain (amino acids 1109-1162, Fig. 2c). This raises the possibility that Tax might interfere with TAX1BP2 dimerization.

Figure S4 Phosphorylation of TAX1BP2. (a) Phosphorylation by Nek2. HEK293T cells were transfected with expression plasmids for HA-TAX1BP2 and Myc-Nek2. The expression of TAX1BP2 and Nek2 was detected with anti-HA and anti-Myc. (b) Cdk2 phosphorylation of TAX1BP2 in culture cells. TAX1BP2 and His-Cdk2 constructs were co-transfected into HEK293T cells and the lysates were probed with anti-HA and antiCdk2 antibodies. Both His-Cdk2 and endogenous Cdk2 were detected. BP2* indicates a slow-migrating species corresponding to hyperphosphorylated TAX1BP2. (c) In vitro phosphorylation of TAX1BP2 by Cdk2. Bacterially produced His-BP2 was incubated with one or two doses of reconstituted Cdk2/cyclin A complex in the presence of $\left[\gamma^{32}\right]$ ATP. The autoradiograph shows the phosphorylation of TAX1BP2 by Cdk2 and histone H1 was included as a control for Cdk2 activity. The TAX1BP2 band is more evident in a longer exposure shown in the lower panel.

Figure S5 Full scan of Western blots. 

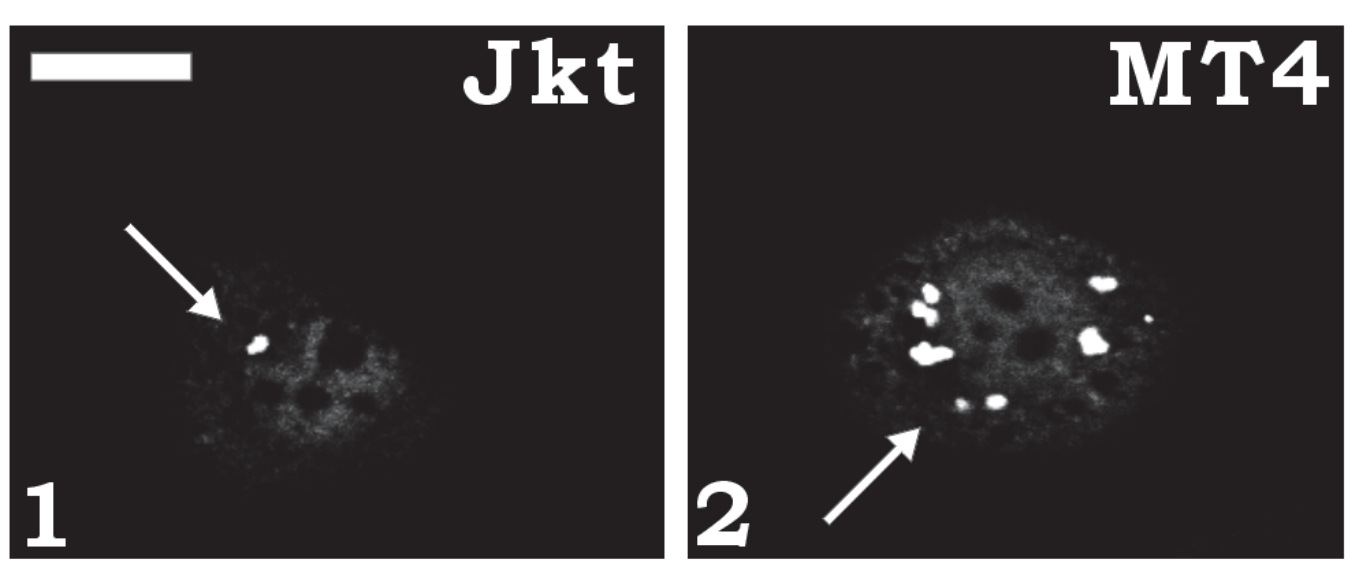

b

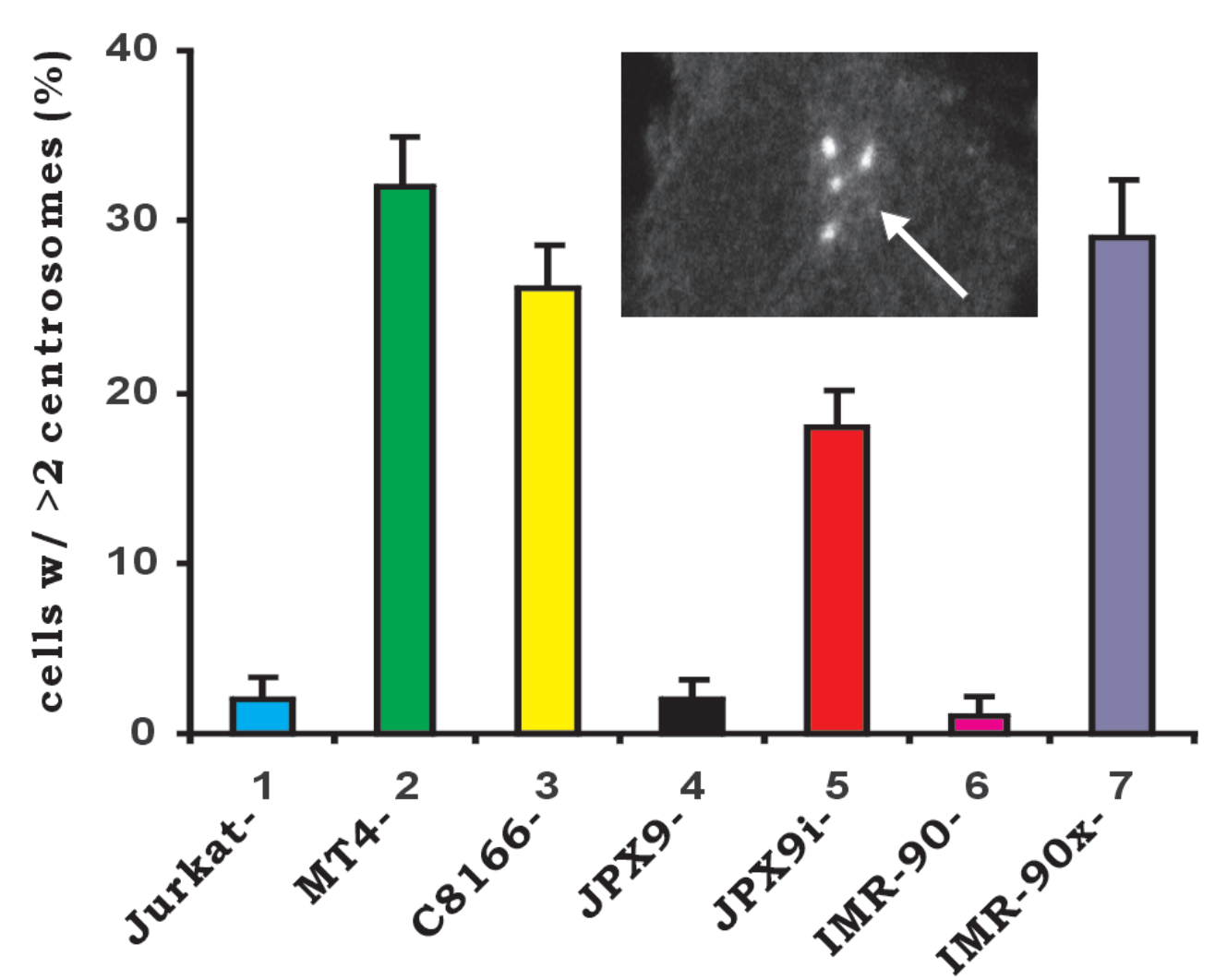

e

\section{Tax}

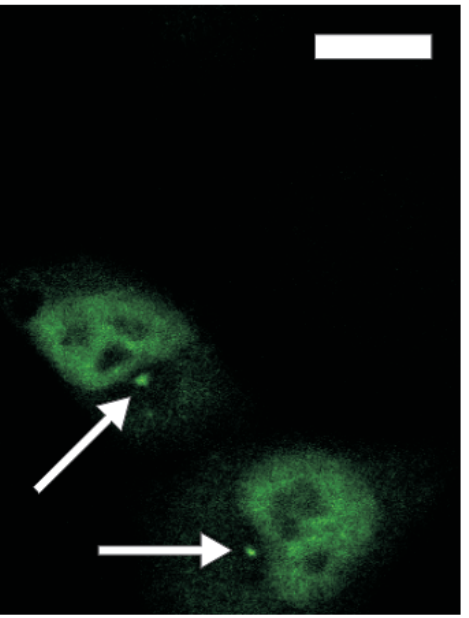

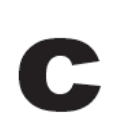

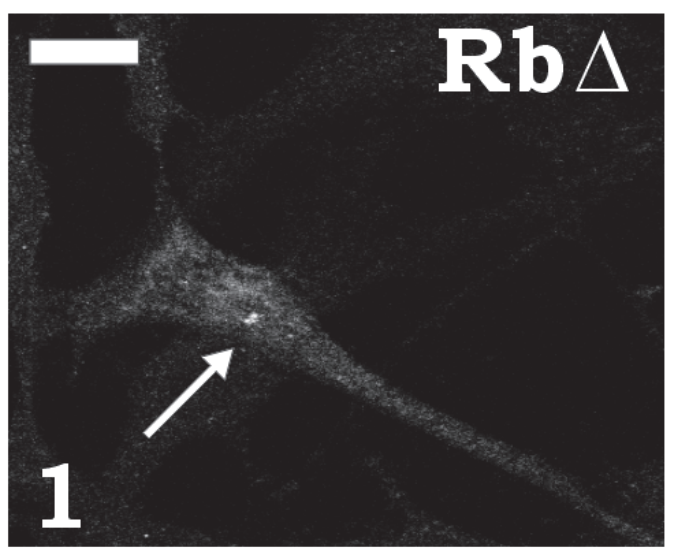

d

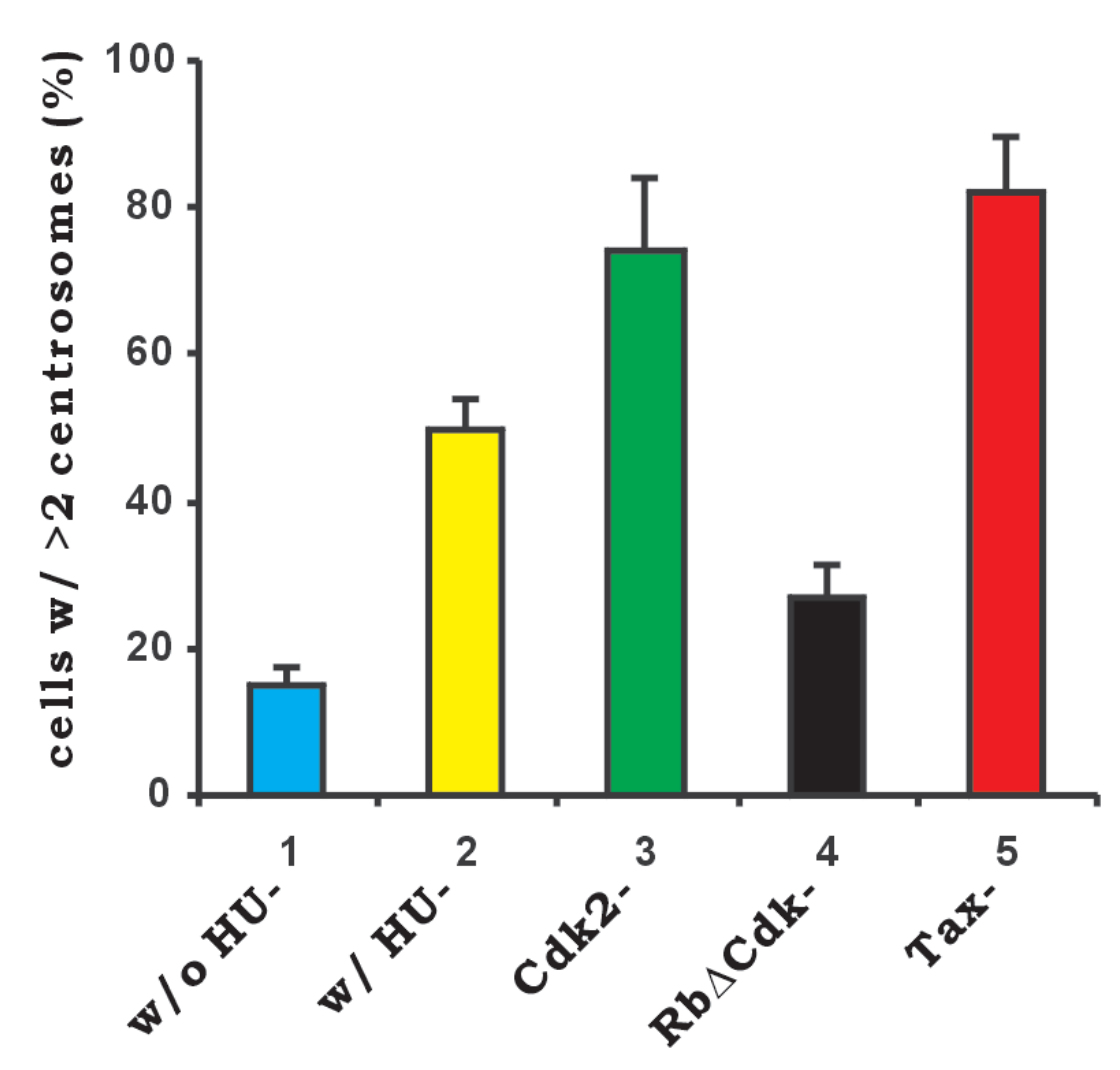

$f$

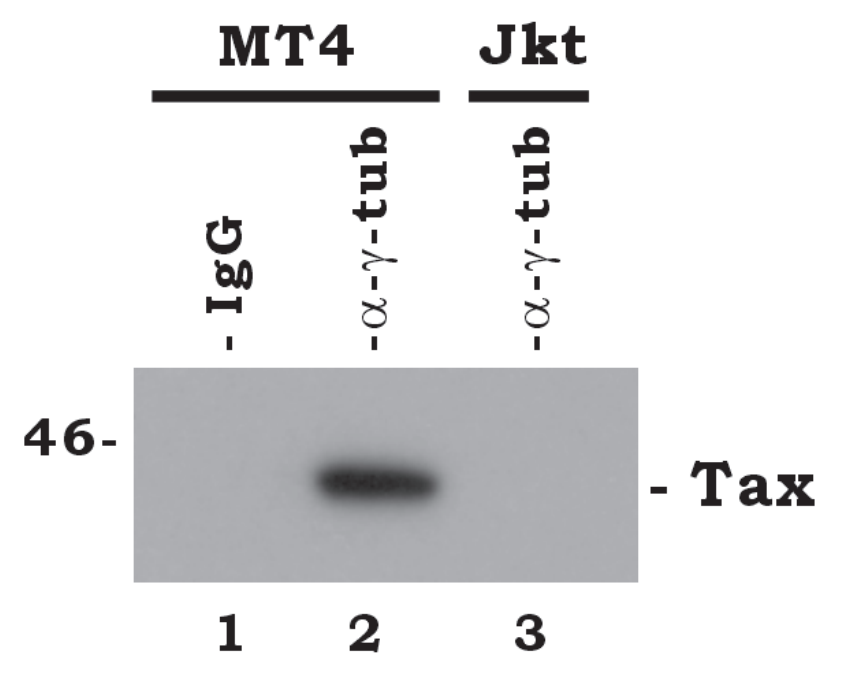


2

BP2

$\gamma($ tubulin)

merge

b
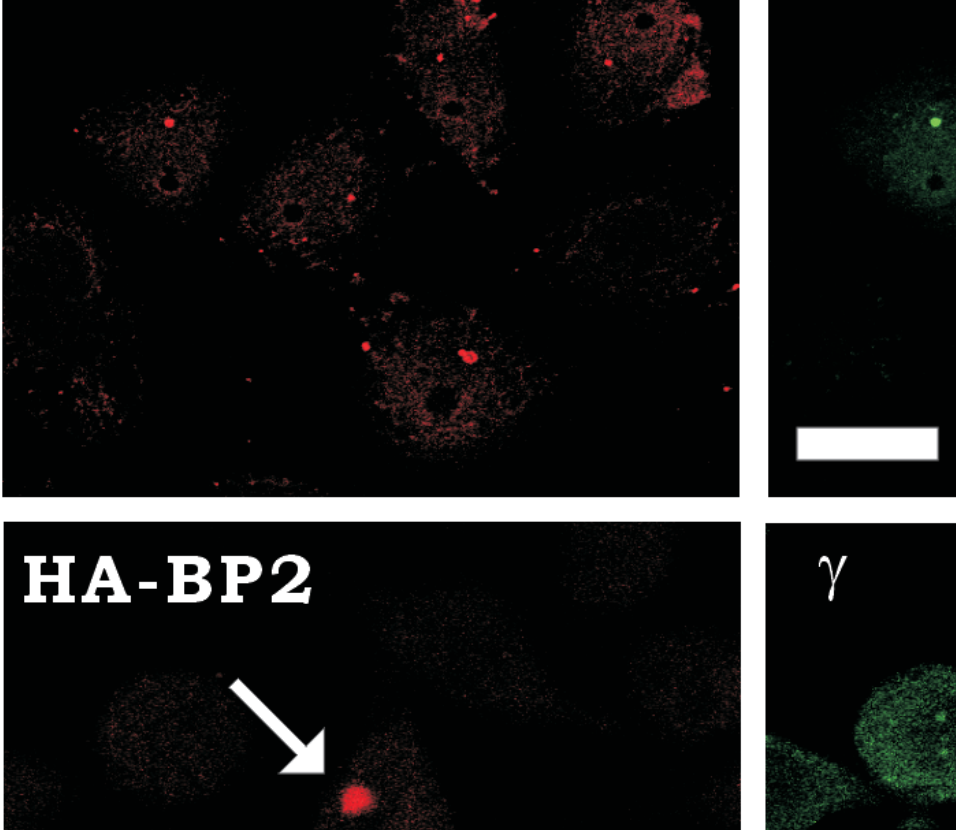

ine

C

M6

e
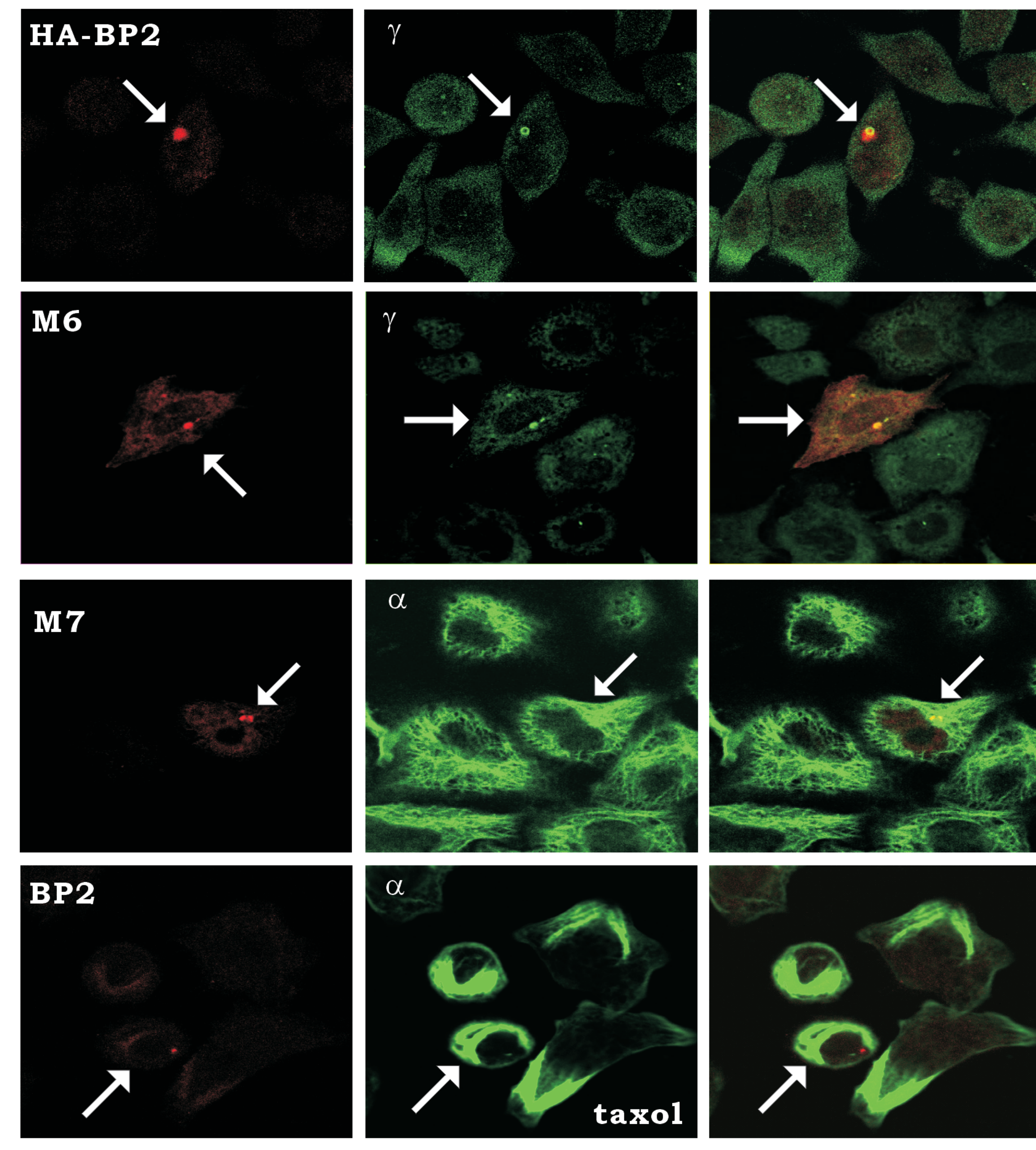

d

M7

f
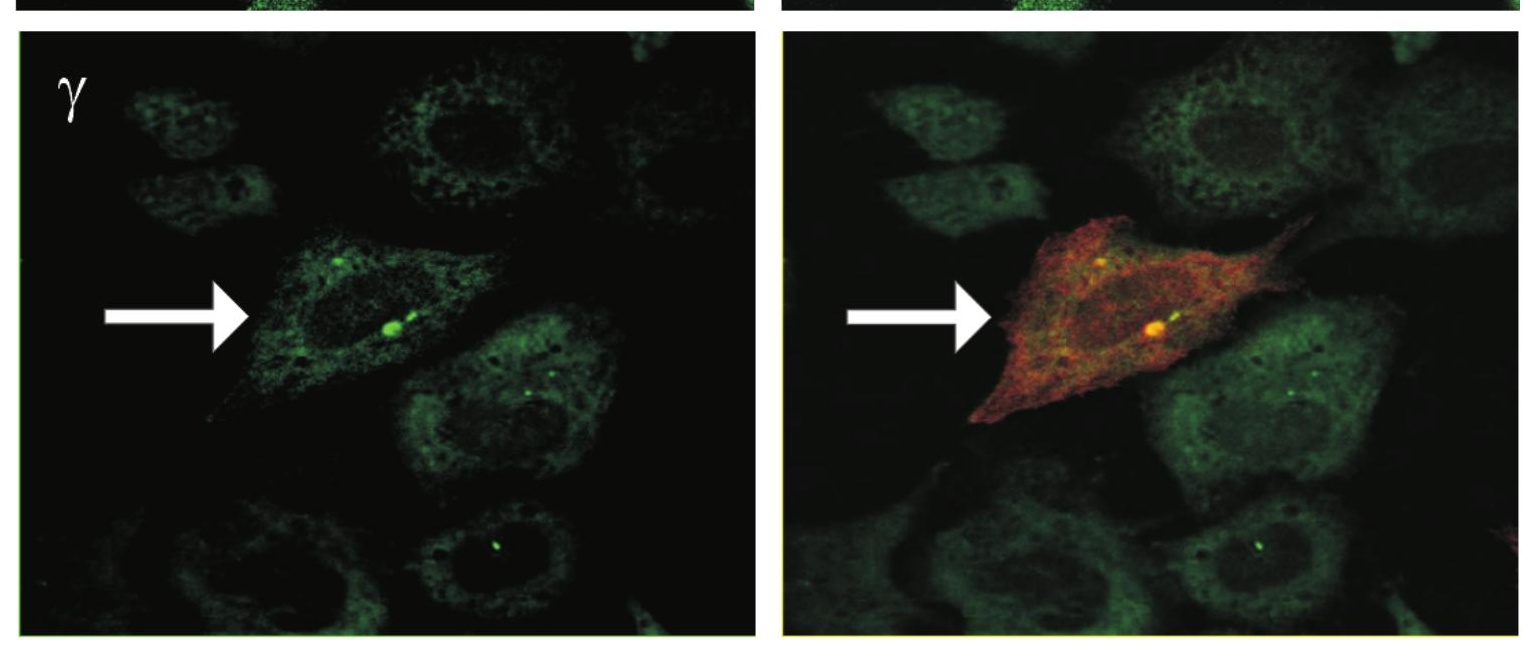

$\nwarrow$
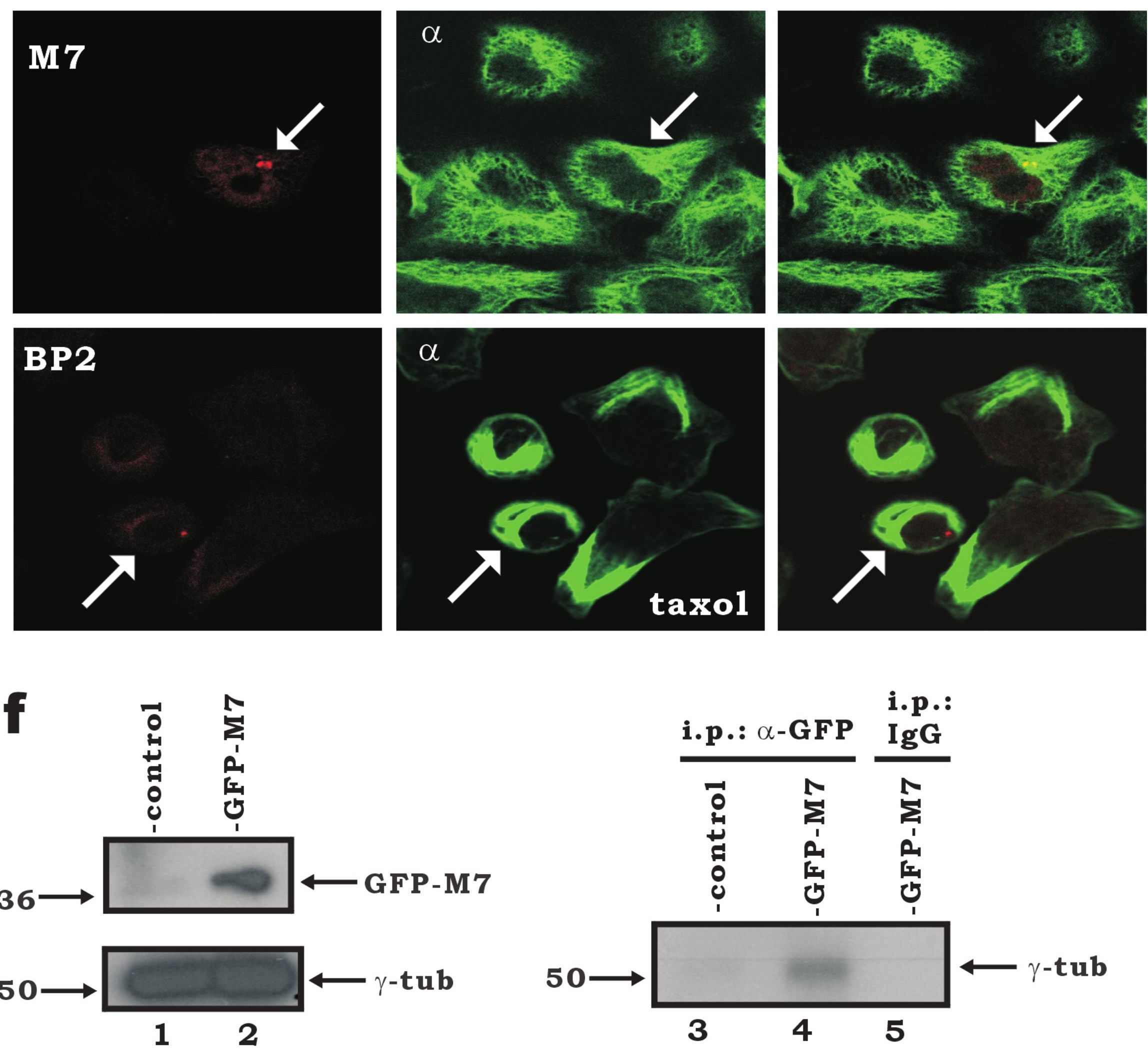

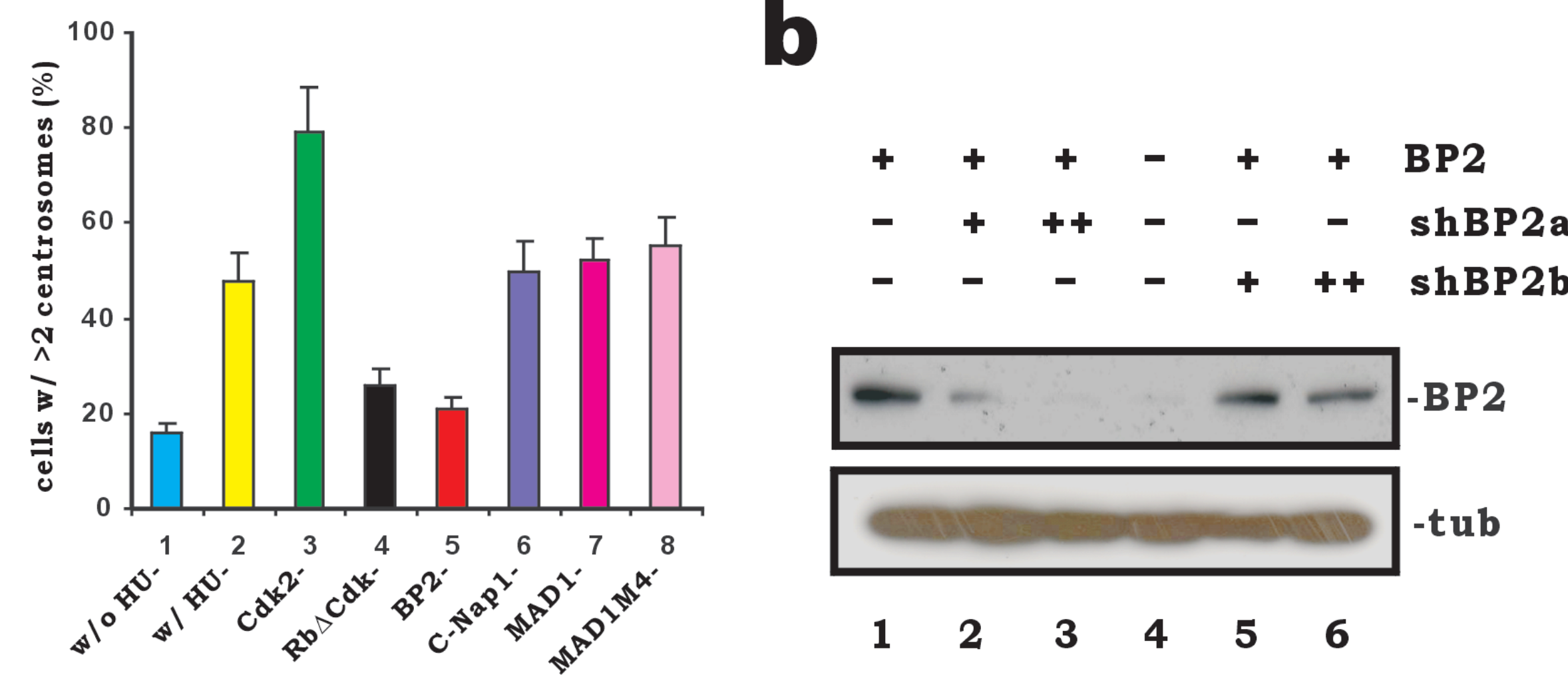

C
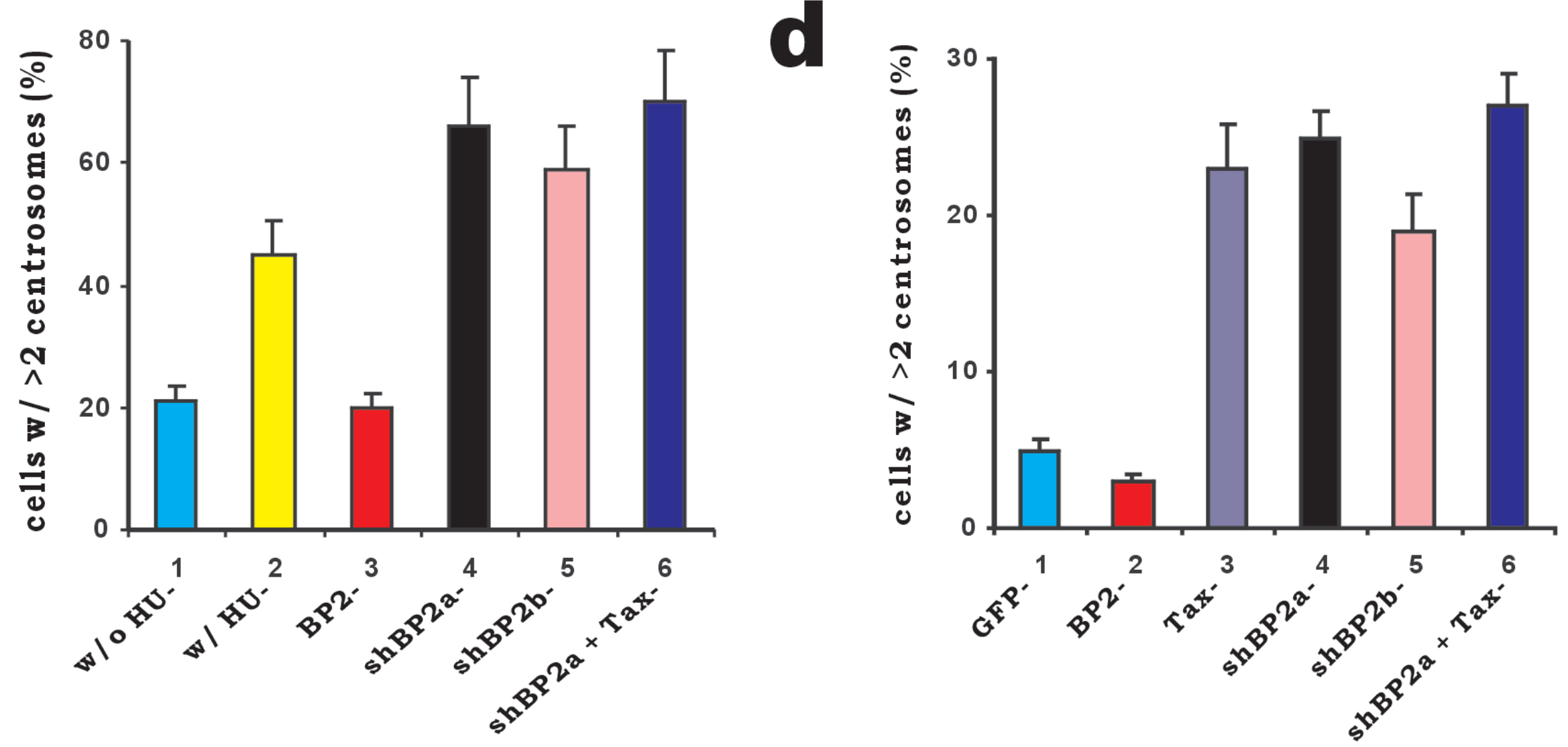

Figure 4 

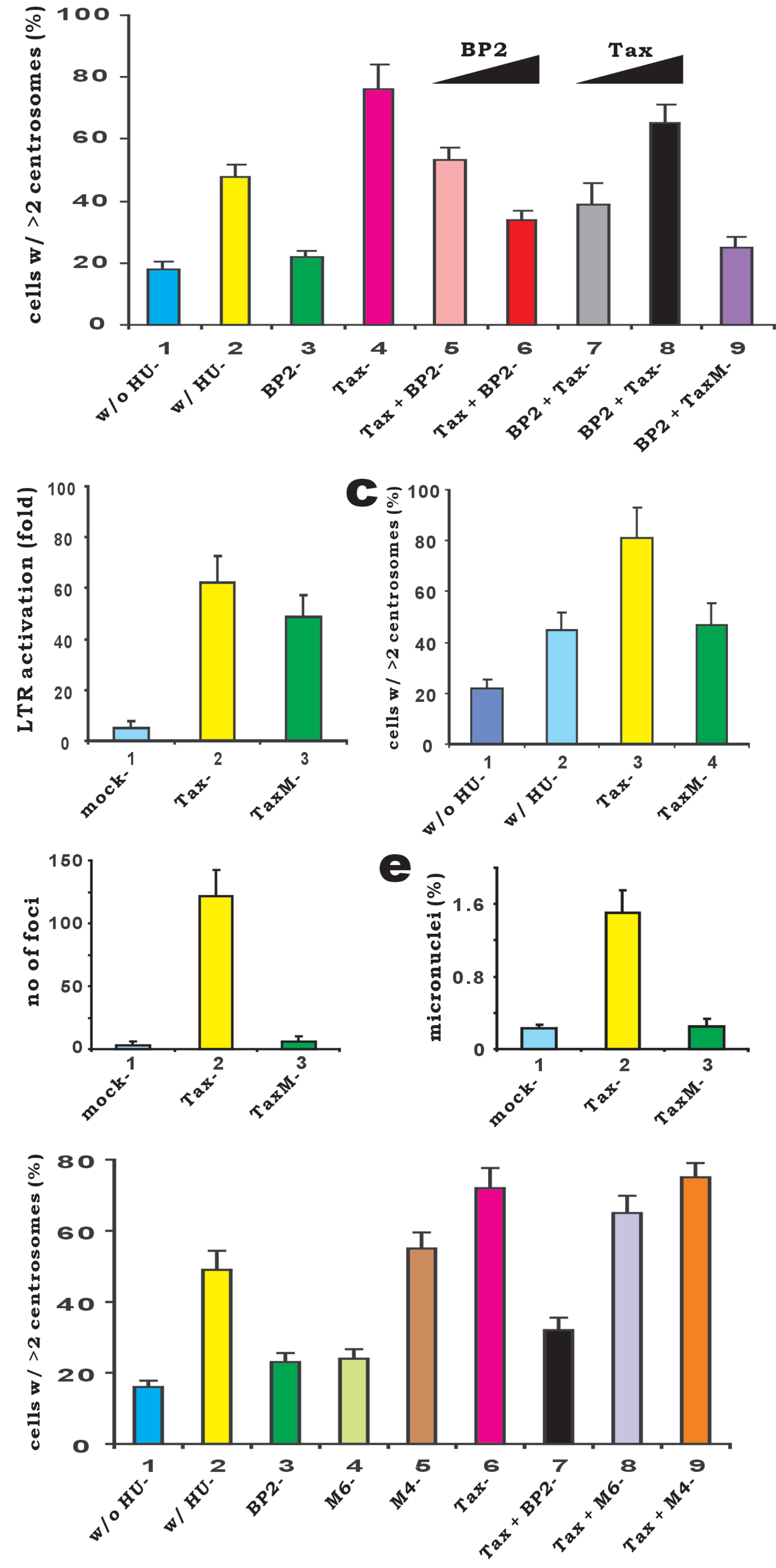

Figure 5 